\title{
Molecular structure effects in the energy loss of swift boron molecular ions in solids
}

\author{
Rafael Garcia-Molina $\dagger$, Santiago Heredia-Avalos $\dagger$ and Isabel Abril $\ddagger$ \\ $\dagger$ Departamento de Física, Universidad de Murcia, Apartado 4021, E-30080 Murcia, Spain \\ \$ Departament de Física Aplicada, Universitat d'Alacant, Apartat 99, E-03080 Alacant, Spain
}

Received 1 February 2000, in final form 14 April 2000

\begin{abstract}
We analyse the effects on the stopping power due to the geometrical structure of swift $\mathrm{B}_{n}^{+}(n=2-6)$ ions incident on amorphous carbon targets. We use the dielectric formalism to describe both the interaction of the projectile with the stopping medium and the vicinage effects on the atomic ions resulting from the dissociation of each $\mathrm{B}_{n}^{+}$ion. The variation of the energy loss with the temporal evolution of the relative positions of the atomic ions, due to Coulomb explosion, has also been taken into account. The calculated stopping power ratio for each $\mathrm{B}_{n}^{+}$ion reproduces fairly well the main trends, as a function of the dwell time, observed in the available experimental data. The differences among the stopping power ratios of the $\mathrm{B}_{n}^{+}$ions disappear as the number of atomic ions, $n$, increases.
\end{abstract}

\section{Introduction}

Important phenomena appear in the interaction of fast molecular ions with solids, which are related to the charge state of the ions [1], the molecular structure [2-4], the modification and damage of materials [5-7], and other interesting processes in atomic collisions in solids.

When a swift molecular ion impinges on a solid, only a few collisions are needed to strip away its binding electrons. The atomic ions resulting from the molecular dissociation travel in a correlated manner under the influence of the mutual Coulomb force and, therefore, they become separated as they advance through the target; this is called Coulomb explosion. The difference between the energy loss of a molecular ion and the sum of the energy losses of its individual constituents was first analysed by Brandt et al [8], and subsequently several theoretical and experimental papers have been published on this [9-16].

Recently, the stopping power of carbon foils for swift $\mathrm{B}_{n}^{+}(n=2-4)$ ions with the energy of $0.8 \mathrm{MeV} /$ atom has been measured [17], and a theoretical work [18] described the electronic energy loss of clusters in solids in an unsatisfactory manner. The purpose of this paper is to analyse the influence of the geometrical structure of individual boron molecules on the vicinage effects that affect the stopping power, for $\mathrm{B}_{n}^{+}(n=2-6)$.

For the range of projectile energies that we will consider (several hundreds of $\mathrm{keV}$ per atom), the nuclear energy loss is negligible compared to the electronic energy loss of the molecular ion, which will be calculated using a model based on the dielectric formalism [19]. The energy lost by the components of the molecular ions after they exit from the foil is obtained as an average of the instantaneous energy loss over the dwell time; in this manner we take into account the time variation of the relative distance between the atomic ions dissociated from the molecular ion, as a result of the Coulomb explosion. The electronic density of the atomic ions 
is described by means of hydrogen-like orbitals, and the average number of electrons bound to each atomic ion is calculated through the degree of ionization as a function of the projectile velocity [20]. We consider a random orientation of the incident boron molecular ion as in the experimental situation that we are going to compare with. Note that we use atomic units in this paper†, except where otherwise stated.

This paper is organized as follows. In section 2 we introduce the model used in our calculations; a presentation of the results and a comparison with the available experimental data is made in section 3. Finally, the conclusions are presented in section 4.

\section{Model}

The energy loss of a molecular ion moving through a solid shows important differences, called vicinage or interference effects, when compared with the energy lost by its individual atomic constituents. The origin of these effects is the interference of the electronic excitations produced in the solid by the correlated motion of the atomic ions. A useful magnitude that quantifies these effects is the instantaneous stopping power ratio, $R_{n}$, which is defined as

$$
R_{n}=\frac{S_{n}}{n S_{1}}
$$

where $S_{n}$ and $S_{1}$ are the stopping powers of the target for the molecular ion and each of its atomic constituents, respectively, and $n$ is the number of atomic ions that form the molecular ion. Note that $R_{n}=1$ means that there are no vicinage effects; $R_{n}>1$ (or $\left.<1\right)$ means an increase (or diminution) of the energy lost by the molecular ion compared with the energy lost by its $n$ uncorrelated constituent atomic ions.

In order to compare the experimental values of the stopping power ratio with our calculated values, we must consider the temporal evolution of the $\mathrm{B}_{n}^{+}$-ion structure during its travel through the solid, averaging the instantaneous stopping power ratio, equation (1), over the dwell time, $\tau=D / v$ :

$$
\left\langle R_{n}\right\rangle=\frac{1}{\tau} \int_{0}^{\tau} \mathrm{d} t R_{n}(t)
$$

where $D$ is the target thickness and $v$ is the molecular ion velocity [21].

We calculate the stopping power ratio for the projectile within the framework of the dielectric formalism, which supposes a linear response of the medium to an external perturbation. In this scheme, the instantaneous stopping power ratio for $n$ correlated atomic ions that travel with velocity $v$ in a target is given by [19]

$$
R_{n}=1+\frac{2}{n} \sum_{i=1}^{n-1} \sum_{j>i}^{n} I\left(r_{i j}\right)
$$

where $I\left(r_{i j}\right)$ is the vicinage function that takes into account the spatial correlation between the atomic ions $i$ and $j$ separated by an interatomic distance $r_{i j}$. The previous equation arises from a superposition of undisturbed wake potentials for the individual atomic ions. For a random orientation of the molecular ion with respect to the beam direction, the vicinage function $I\left(r_{i j}\right)$ is given by

$I\left(r_{i j}\right)=\frac{2}{\pi v^{2} S_{1}} \int_{0}^{\infty} \frac{\mathrm{d} k}{k}[Z-\rho(k)]^{2} \frac{\sin \left(k r_{i j}\right)}{k r_{i j}} \int_{0}^{k v} \mathrm{~d} \omega \omega \operatorname{Im}\left[\frac{-1}{\epsilon(k, \omega)}\right]$

$\dagger$ Atomic units are defined by the condition $m_{e}=e=\hbar=1$, where $m_{e}$ is the mass of the electron and $e$ is the elementary charge. 
where $k$ and $\omega$ are, respectively, the momentum and energy transferred to the excitations of the target electrons; $Z$ is the atomic number of each atomic ion and $\rho(k)$ is the Fourier transform of its electronic density; $\operatorname{Im}[-1 / \epsilon(k, \omega)]$ is the energy-loss function (ELF) of the target, which describes its response to an external perturbation. We have used a sum of Mermin-type ELF [22] in order to obtain a realistic description of the amorphous carbon ELF [23,24]. We include the contribution to the energy loss of the outer electrons as well as that of the electrons from the atomic inner shells - that is, the full spectrum of excitations. More details about this procedure and the fitting parameters used for the amorphous carbon ELF are given in reference [24]. The stopping power for a boron atomic ion, $S_{1}$, was also calculated using the dielectric formalism:

$$
S_{1}=\frac{2}{\pi v^{2}} \int_{0}^{\infty} \frac{\mathrm{d} k}{k}[Z-\rho(k)]^{2} \int_{0}^{k v} \mathrm{~d} \omega \omega \operatorname{Im}\left[\frac{-1}{\epsilon(k, \omega)}\right] .
$$

We have used the $1 \mathrm{~s}$ and 2 s hydrogen-like orbitals to describe the electronic density of the boron atomic ions. The corresponding Fourier transform, $\rho(k)$, can be written in a compact form as follows:

$$
\begin{aligned}
\rho(k)=[N \Theta(2-N)+2 \Theta(N-2)]\left[1+\left(\frac{k}{2 Z_{\mathrm{S}}}\right)^{2}\right]^{-2} \\
-2(N-2) \Theta(N-2)\left[\left(\frac{k}{Z_{\mathrm{S}}}\right)^{2}-1\right]\left[\left(\frac{k}{Z_{\mathrm{S}}}\right)^{2}-\frac{1}{2}\right]\left[1+\left(\frac{k}{2 Z_{\mathrm{S}}}\right)^{2}\right]^{-4}
\end{aligned}
$$

where $\Theta(\cdots)$ is the Heaviside step function. In the above expression, $N$ is the average number of electrons bounded in the $1 \mathrm{~s}$ and $2 \mathrm{~s}$ atomic orbitals, which depends [20] on the projectile velocity (note that $N<4$ when the projectile energy is $0.8 \mathrm{MeV} /$ atom), and $Z_{\mathrm{S}}$ is the effective nuclear charge given by Slater's rules [25]. This model is, in a first approximation, a good description of the electronic density of an atomic ion and, for the low value of $N$ involved in our calculations, it is better than the general treatment proposed in reference [26].

The stopping power ratio for molecular ions depends on the geometrical arrangement of the atomic ions that form the molecule. In this work we consider the molecular ions from $\mathrm{B}_{2}^{+}$to $\mathrm{B}_{6}^{+}$, whose geometrical structures were solved by means of $a b$ initio calculations in references $[27,28]$. The results obtained in these works are rather similar, and they indicate planar or quasi-planar geometries for the $\mathrm{B}_{n}^{+}(n=2-6)$ ions; the corresponding boron molecular geometries appear as insets in figures $1(\mathrm{a})$ and $1(\mathrm{~b}) . \mathrm{B}_{2}^{+}$is a linear molecule with a bond length of $4.16 \mathrm{au}, \mathrm{B}_{3}^{+}$is an equilateral triangle with a bond length of $2.93 \mathrm{au}$, and $\mathrm{B}_{4}^{+}$is a rhombus where the bond length is 2.91 au, and their bond angles are $89.92^{\circ}, 90.08^{\circ}$, and $89.92^{\circ}$ respectively; for simplicity, we will suppose here that the $\mathrm{B}_{4}^{+}$ion is a perfect square. $\mathrm{B}_{5}^{+}$is a slightly deformed pentagon where the average bond length is $2.95 \mathrm{au}$; in our calculations we assume it to be a regular pentagon since the three bond lengths differ by only $0.002 \mathrm{au}$. Finally, $\mathrm{B}_{6}^{+}$is a distorted hexagon where the distance between the centre of the ring and the two atoms along the larger axis is $3.78 \mathrm{au}$, the four lateral atoms are at 2.40 au from the centre, and the angle between the centre and two inequivalent atoms is $52.0^{\circ}$.

Due to the symmetry of the initial structures of the $\mathrm{B}_{n}^{+}(n=2-5)$ ions, the Coulomb explosion changes only the molecule size, preserving their initial shapes. For this reason, one single variable is enough to characterize the temporal evolution of each molecular structure, and we choose the interatomic distance between the nearest atomic neighbours, $r_{n}$. However, $\mathrm{B}_{6}^{+}$is not a symmetrical hexagon and, therefore, the Coulomb explosion slightly changes the molecule shape; in this case, we describe the temporal evolution of the $\mathrm{B}_{6}^{+}$structure by means of a set of distances, $r_{i j}$, between the atomic ions $i$ and $j$.

In order to obtain the temporal evolution of the interatomic distances between the $n$ boron atomic ions, we solve numerically the set of coupled Newton equations for these atomic ions, 


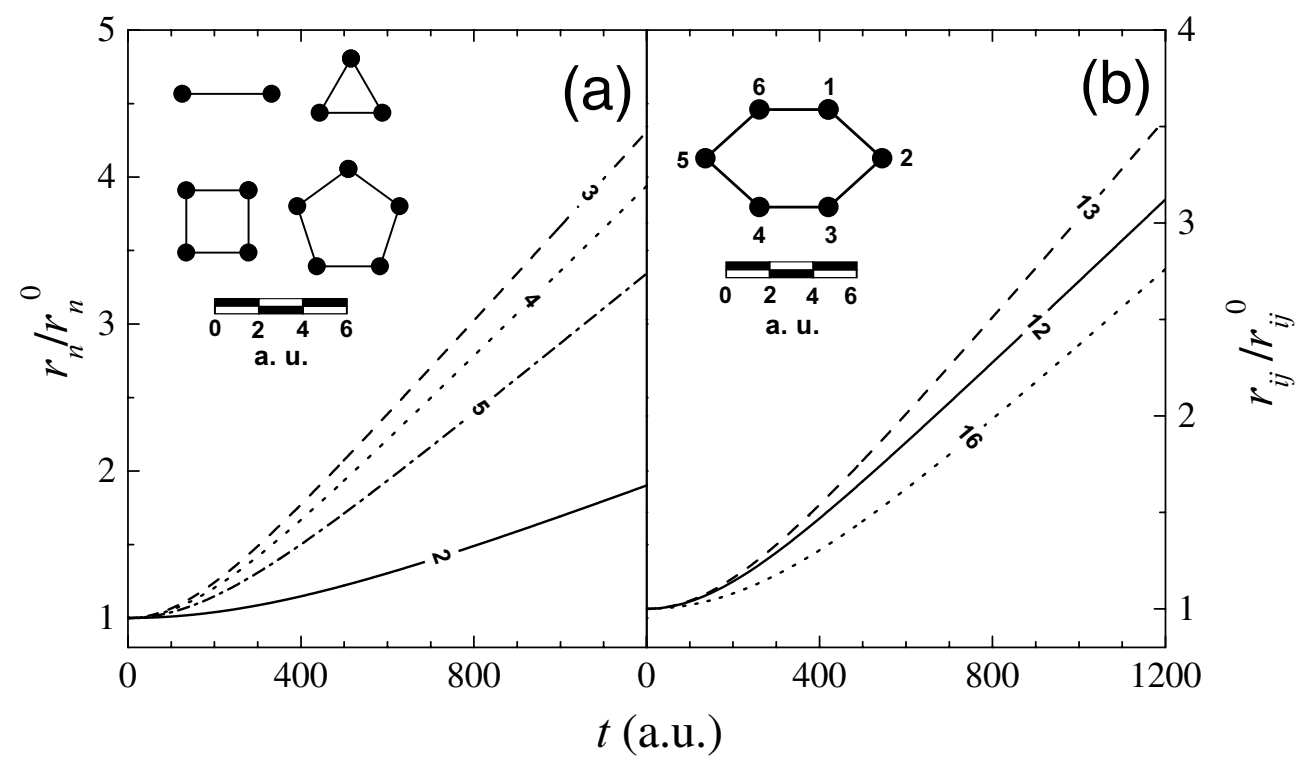

Figure 1. The temporal evolution of the normalized interatomic distances between the atomic constituents of the boron molecular ions moving through amorphous carbon: (a) $r_{n} / r_{n}^{0}$ for $\mathrm{B}_{n}^{+}$ $(n=2-5)$ and (b) $r_{i j} / r_{i j}^{0}$ for $\mathrm{B}_{6}^{+}$. The numerical labels on the curves in (a) and (b) indicate $n$ and $i j$, respectively. The projectile energy is $0.8 \mathrm{MeV} /$ atom. The insets show the $\mathrm{B}_{n}^{+}$structures. $r_{2}^{0}=4.16 \mathrm{au}, r_{3}^{0}=2.93 \mathrm{au}, r_{4}^{0}=2.91 \mathrm{au}, r_{12}^{0}=2.98 \mathrm{au}, r_{13}^{0}=3.78 \mathrm{au}$, and $r_{16}^{0}=2.96 \mathrm{au}$.

each with a charge $Z^{\prime}=Z-N$, interacting through a screened Coulomb potential. For any pair of atomic ions separated an interatomic distance $r_{i j}$, this potential is given by

$$
V\left(r_{i j}\right)=\frac{Z^{\prime}}{r_{i j}} \exp \left(-\frac{r_{i j}}{a}\right)
$$

where the screening length is $a=v / \omega_{\mathrm{p}}$ if $v>v_{\mathrm{F}}$ and $a=v_{\mathrm{F}} /\left(3^{1 / 2} \omega_{\mathrm{p}}\right)$ otherwise [29]; $\omega_{\mathrm{p}}$ $(=0.945 \mathrm{au})$ is the plasmon energy and $v_{\mathrm{F}}(=1.2 \mathrm{au})$ is the Fermi velocity for amorphous carbon.

\section{Results and discussion}

Figure 1(a) shows the interatomic distance $r_{n}$ (normalized to the initial interatomic distance $r_{n}^{0}$ between the nearest atomic neighbours) as a function of the time after the molecule enters into the target, when the $\mathrm{B}_{n}^{+}$energy is $0.8 \mathrm{MeV} /$ atom; solid, dashed, dotted, and dot-dashed lines correspond to $n=2,3,4$, and 5, respectively. We found that, as a consequence of the geometrical arrangement of the atoms in the molecular ion, $\mathrm{B}_{3}^{+}$experiences a bigger repulsion force than $\mathrm{B}_{4}^{+}$, despite the fact that $r_{4}^{0}<r_{3}^{0}$. Figure 1(b) shows the temporal evolution of three typical interatomic distances of the $\mathrm{B}_{6}^{+}$ion when the projectile energy is $0.8 \mathrm{MeV} / \mathrm{atom}$; solid, dashed, and dotted lines correspond to $r_{12}, r_{13}$, and $r_{16}$, respectively.

By means of the relationship between the time, $t$, and $r_{n}$ (for $\mathrm{B}_{n}^{+}(n=2-5)$ ) or $r_{i j}$ (for $\left.\mathrm{B}_{6}^{+}\right)$, we can obtain the vicinage function, $I\left(r_{n}\right)$ or $I\left(r_{i j}\right)$, corresponding to each instantaneous geometrical arrangement (see equation (4)). In figures 2(a) and 2(b), we present the temporal evolution of the vicinage function for $\mathrm{B}_{n}^{+}(n=2-5)$ ions and $\mathrm{B}_{6}^{+}$ions, respectively, when the projectile energy is $0.8 \mathrm{MeV} /$ atom. The vicinage function has positive and negative values, 


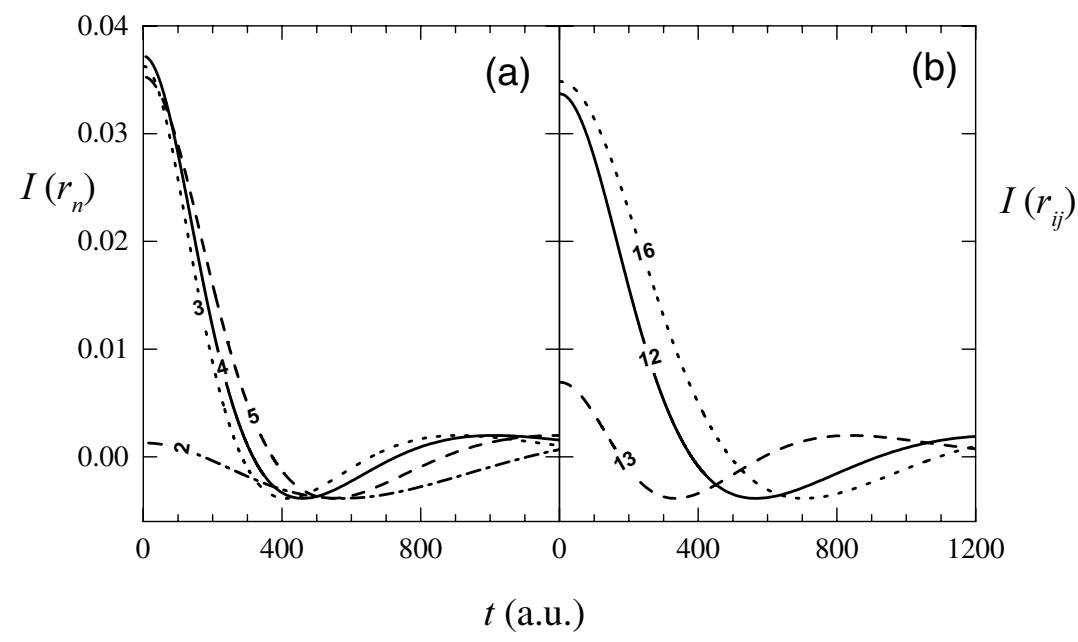

Figure 2. The temporal evolution of the vicinage function for the $\mathrm{B}_{n}^{+}$ions moving through amorphous carbon. The numerical labels on the curves in (a) and (b) indicate $n$ and $i j$, respectively. The projectile energy is $0.8 \mathrm{MeV} /$ atom.

depending on the interatomic distances, because the force due to the wake potential of the leading ion acting on the trailing ones may be repulsive or attractive [24]. The vicinage function $I\left(r_{n}\right)$ is rather similar for $\mathrm{B}_{n}^{+}(n=3-5)$ ions, because the initial interatomic distances $r_{n}^{0}$ between the nearest atomic neighbours do not differ appreciably. However, the vicinage function for $\mathrm{B}_{2}^{+}$is the smallest one, because $r_{2}^{0}>r_{n}^{0}(n=3-5)$, and the vicinage effects decrease for larger distances. Figure 2(b) shows the vicinage function for three representative interatomic distances of the $\mathrm{B}_{6}^{+}$ion: $I\left(r_{12}\right), I\left(r_{13}\right)$, and $I\left(r_{16}\right)$; it is important to note that the interference effects can cancel out in some cases, because the vicinage function for a given time could be negative for two atomic ions and positive for the other pair of atomic ions.

Using the previous values of the vicinage function as a function of time, we have evaluated the average stopping power ratio $\left\langle R_{n}\right\rangle$ according to equations (2) and (3). This $\left\langle R_{n}\right\rangle$ is the magnitude to be compared with the experimental data [17] obtained after the constituents of the molecular ions leave the amorphous carbon foil.

Figure 3 shows the average stopping power ratio versus the dwell time, $\tau$, for the case of $\mathrm{B}_{n}^{+}(n=2-6)$ ions incident with an energy of $0.8 \mathrm{MeV} /$ atom on amorphous carbon foils. The symbols in figure 3 represent the experimental stopping power ratio with the corresponding error bars (rather larger for small values of $\tau$ ) [17]; these data seem to suggest that $\left\langle R_{n}\right\rangle>1$, although $\left\langle R_{n}\right\rangle=1$ should also be consistent with the experimental results. The thick lines (labelled with the numbers of molecular constituents) show our calculation results and the thin solid lines (in figures 3(b) and 3(c)) show the calculation results obtained by Kaneko [18]. Our results show that $\left\langle R_{n}\right\rangle$ is always greater than unity, as the experimental results suggest; however, the average stopping power ratio obtained by Kaneko [18] is equal to or smaller than unity even for thinner foils; this is opposite to the trends shown by the experimental points. A possible explanation of these differences is that different energy-loss functions were used in each of the theoretical treatments. In our calculations the maximum vicinage effects in the average stopping power ratio are produced at smaller dwell times (thinner foils) when the boron atomic ions remain closer. At large dwell times the average stopping power ratio goes to one, since the Coulomb explosion of the molecular ion appreciably separates the atomic ions, which then travel in a non-correlated way through the target for a longer time. This behaviour 


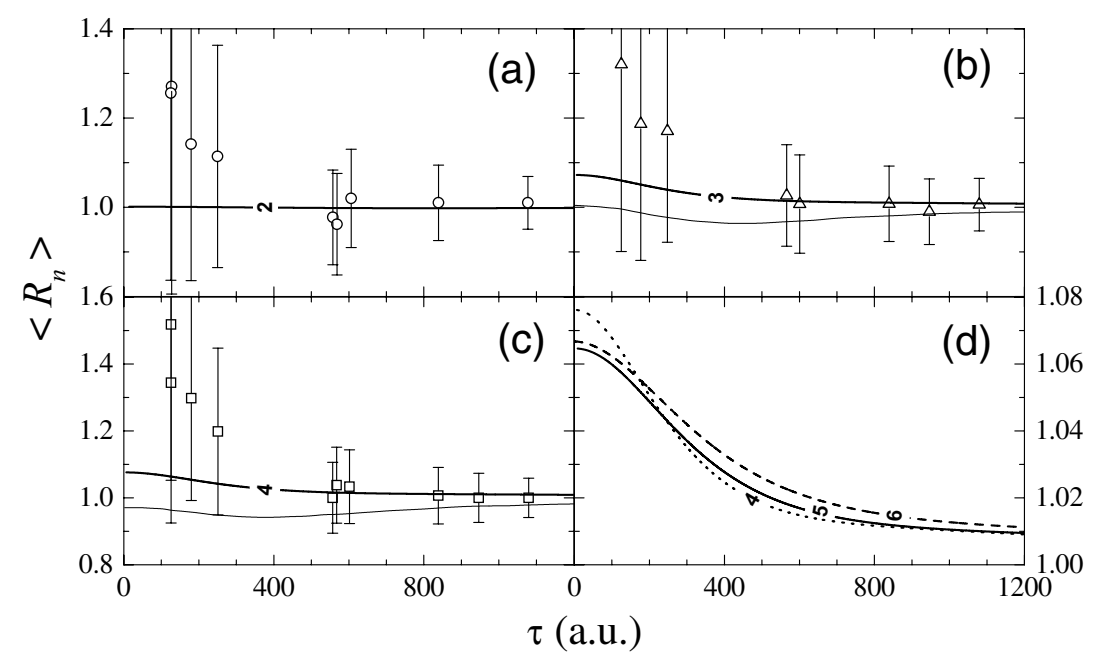

Figure 3. Stopping power ratio as a function of dwell time for the $\mathrm{B}_{n}^{+}$ions after travelling through an amorphous carbon foil. The thick lines (with the numerical labels indicating the value of $n$ ) show our calculation results, the thin solid lines show the theoretical results obtained by Kaneko [18], and the symbols represent the experimental data [17]. The projectile energy is $0.8 \mathrm{MeV} /$ atom.

agrees satisfactorily with the trends shown by the experimental data for all molecular ions.

We obtain that the average stopping power ratio increases with the molecular size for the $\mathrm{B}_{n}^{+}(n=2-4)$ ions, as can be seen in figures 3(a)-3(c). This is due to the contribution of two factors related to the molecular structure: (i) the increase of the number $n$ of atomic ions that constitute the molecular ion, and (ii) the decrease of the initial interatomic distances, $r_{n}^{0}$. The former means that more vicinage effects are expected because more ions are interacting; the latter corresponds to a decrease of the initial interatomic distances $\left(r_{2}^{0}>r_{3}^{0}>r_{4}^{0}\right)$, which also contributes to the increase of the vicinage effects; consequently, the stopping power ratio rises as $n$ goes from 2 to 4 . Moreover, $\left\langle R_{2}\right\rangle$ is practically constant and almost equal to unity for all foil thicknesses, which is due to the large value of $r_{2}^{0}$. However, for the case of $\mathrm{B}_{n}^{+}(n=4-6)$ ions, figure 3(d) shows that there are no important differences between their average stopping power ratios, although now the number of ions increases. These small values of $\left\langle R_{n}\right\rangle$ for $n=4-6$ and the absence of a significant variation in their behaviour could be explained in terms of cancellations of the vicinage effects due to the presence of several atomic ions at different distances from a given atomic ion.

\section{Conclusions}

We have discussed the influence of the molecular structure on the energy loss of boron molecular ions travelling through amorphous carbon foils. The electronic excitations (due to the valence and inner-shell electrons) induced in the solid are described by the dielectric formalism, using a realistic description of the energy-loss function. Moreover, we include the electronic density of the atomic ions using hydrogen-like orbitals with the average number of electrons bound to each atomic ion, which is a function of the projectile velocity. In order to evaluate the temporal evolution of the molecular geometry, we have considered the Coulomb explosion of the atomic ions that compose the molecular ion.

The calculated average stopping power ratio for $0.8 \mathrm{MeV} /$ atom $\mathrm{B}_{n}^{+}(n=2-6)$ ions has 
been compared with experimental data [17], as a function of the dwell time. Calculations and experiments agree fairly well.

We conclude that the number of atoms that form the molecular ion, as well as their geometrical distribution, affect the stopping power ratio for the boron molecular ions; we find an increment in the stopping power ratio as the molecular size of the $\mathrm{B}_{n}^{+}(n=2-4)$ ions increases. However, this tendency does not continue for the larger molecular ions, $\mathrm{B}_{n}^{+}$ ( $n=4-6)$, due to the cancellation of the vicinage effects due to the different interatomic distances of the atomic ions.

The work presented is based on a first-order perturbation theory and it does not consider the Barkas and Bloch terms. The contribution to the stopping power due to the first of these is $\sim 5 \%$ for the case studied here [30]; therefore, the inclusion of the Barkas term would probably not significantly affect our calculations, i.e., the stopping power ratio would remain greater than unity and inside the experimental error bars. However, the Barkas term should be included when more accurate calculations of the stopping power and the vicinage function are desired, in order to allow comparison with experimental data with smaller errors bars. On the other hand, the Bloch term - the other correction - should be taken into account in an improved model; recent calculations [31] suggest that the inclusion of the Bloch correction would increase our values of the stopping power ratio by about $20 \%$, which would make them in agreement with the experimental data.

\section{Acknowledgments}

This work was supported by the Spanish Dirección General de Enseñanza Superior (project PB96-1118) and by the Generalitat Valenciana (project GV99-54-1-01). SHA thanks the Spanish Ministerio de Educación y Cultura for a research grant. We thank T Kaneko for providing us with a copy of his work prior to publication.

\section{References}

[1] Maor D, Cooney P J, Faibis A, Kanter E P, Koening W and Zabransky B J 1985 Phys. Rev. A 32105

[2] Gemmell D S 1980 Chem. Rev. B 18301

[3] Kella D, Algranati H, Feldman H, Herber O, Kovner H, Malkin E, Miklazky E, Naaman R, Zajfman D, Zajfman $\mathrm{J}$ and Vager Z 1993 Nucl. Instrum. Methods A 329440

[4] Jakas M M and Capuj N E 1995 J. Phys.: Condens. Matter 74593

[5] Knauer W and Poeschel R L 1988 J. Vac. Sci. Technol. B 6456

[6] Salehpour M, Fishel D L and Hunt J E 1988 Phys. Rev. B 3812320

[7] Jensen J, Dunlop A, Della-Negra S and Pascard H 1998 Nucl. Instrum. Methods B 135295

[8] Brandt W, Ratkowski A and Ritchie R H 1974 Phys. Rev. Lett. 331325

[9] Tape J W, Gibson W M, Remillieux J, Laubert R and Wegner H E 1976 Nucl. Instrum. Methods 13275

[10] Steuer M F, Gemmell D S, Kanter E P, Johnson E A and Zabransky B J 1982 Nucl. Instrum. Methods B 194277

[11] Basbas G and Ritchie R H 1982 Phys. Rev. A 251943

[12] Rule D W and Crawford O H 1984 Phys. Rev. Lett. 52934

[13] Kemmler J, Koschar P, Burkhard M and Groeneveld K O 1985 Nucl. Instrum. Methods B 1262

[14] Ashley J C and Echenique P M 1987 Phys. Rev. B 358701

[15] Nagy I, Arnau A and Echenique P M 1990 Nucl. Instrum. Methods B 4847

[16] Ben-Hamu D, Baer A, Feldman H, Levin J, Heber O, Amitay Z, Vager Z and Zajfman D 1997 Phys. Rev. A 56 4786

[17] Narumi K, Nakajima K, Kimura K, Mannami M, Saitoh Y, Yamamoto S, Aoki Y and Naramoto H 1998 Nucl. Instrum. Methods B $\mathbf{1 3 5} 77$

[18] Kaneko T 1999 Nucl. Instrum. Methods B 15315

[19] Arista N R 1978 Phys. Rev. B 181 
[20] Ziegler J F, Biersack J P and Littmark U 1985 The stopping and range of ions in solids Stopping and Ranges of Ions in Solids vol 1 (New York: Pergamon)

[21] Denton C D, Pérez-Pérez F J, Abril I, Garcia-Molina R and Arista N R 1996 Europhys. Lett. 35499

[22] Mermin N D 1970 Phys. Rev. B 12362

[23] Planes D J, Garcia-Molina R, Abril I and Arista N R 1996 J. Electron Spectrosc. Relat. Phenom. 8223

[24] Abril I, Garcia-Molina R, Denton C D, Pérez-Pérez F J and Arista N R 1998 Phys. Rev. A 58357

[25] Slater J C 1930 Phys. Rev. 3657

[26] Brandt W and Kitagawa M 1982 Phys. Rev. B 255631

[27] Sowa-Resat M B, Smolanoff J, Lapicki A and Anderson S L 1997 J. Chem. Phys. 1069511

[28] Ricca A and Bauschlicher C W Jr 1996 Chem. Phys. 208233

[29] Brandt W 1975 Atomic Collisions in Solids ed S Datz et al (New York: Plenum) p 261

[30] Balashov V V 1997 Interaction of Particles and Radiation with Matter (Berlin: Springer) p 83

[31] Schiwietz G and Grande P L 1999 Nucl. Instrum. Methods B 1531 